\title{
Heart involvement in systemic sclerosis: an ultrasonic tissue characterisation study
}

\author{
Clodoveo Ferri, Vitantonio Di Bello, Antonella Martini, Davide Giorgi, \\ Franca A A Storino, Massimiliano Bianchi, Alessio Bertini, Marco Paterni, \\ Costantino Giusti, Giampiero Pasero
}

Rheumatology Unit, Department of Internal Medicine, University of Pisa, Italy

C Ferri

V Di Bello

A Martini

D Giorgi

F A A Storino

M Bianchi

A Bertini

C Giusti

G Pasero

Institute of Clinical Physiology, CNR, Pisa, Italy

M Paterni

Correspondence to: Professor C Ferri, Rheumatology Unit, Department of Interna Medicine, Via Roma 67, 56100 Pisa, Italy.

Accepted for publication 10 March 1998

\begin{abstract}
Background-Clinicoepidemiological findings indicate that symptomatic heart involvement in patients with systemic sclerosis (SSc) predicts a very poor prognosis. At necropsy studies, SSc heart involvement without significant coronary lesions is characterised by patchy myocyte necrosis and contraction band necrosis with collagen replacement leading to myocardial fibrosis. There is a discrepancy between the frequency of clinically evident myocardial disease $(25 \%)$ and autoptical myocardial fibrosis (81\%).
\end{abstract}

Objective-The aim of this study was to detect preclinical myocardial alterations in SSc patients by ultrasonic videodensitometric analysis.

Methods-Thirty five SSc patients (three male, aged 48.6 (11) SD years, range 22-65) with normal ventricular function and 25 age and sex matched healthy controls were studied. All patients had a negative maximal exercise stress; in all cases arterial hypertension, renal involvement, and diabetes were excluded. Echocardiographic images were digitised by a real time videodigitiser (Tomtec Imaging Systems). Quantitative texture analysis was performed on data from the septum and the posterior wall, obtaining mean gray level histogram (MGL) at both end-diastole (d) and end-systole (s). The cyclic variation index (CVI), was calculated according to the formula ((MGLd-MGLs)/MGLd) $\times$ 100. Left ventricular mass (LVM), body surface corrected, was calculated according to Penn convention.

Results-Comparable systolic and diastolic blood pressure, LVM, diastolic and systolic function were recorded in both SSc patients and controls. In contrast, in SSc patients the CVI, which is the expression of the intrinsic myocardial structural function, was significantly lower than in controls (septum: $-18(28) \%$ v $35(10) \%$, p<0.0001; and posterior wall: $-13(32) \% v$ $50(20) \%, p<0.0001)$. Changes in cyclic echo amplitude, probably related to myocardial fibrosis, were detected in the large majority of SSc patients $(88 \%)$.

Conclusions-Ultrasonic videodensitometric analysis represents a non-invasive, feasible method that can detect early myocardial changes in SSc patients, which could be related to both fibrosis and microcirculatory abnormalities. Their potential evolution towards ventricular dysfunction and their link with cardiac sudden death, because of severe conduction system or rhythm disturbancies, should be further investigated.

(Ann Rheum Dis 1998;57:296-302)

Systemic sclerosis ( $\mathrm{SSc}$ ) is a connective tissue disease characterised by fibrosis of multiple target organs including the skin, lung, gastrointestinal tract, kidney, and heart. ${ }^{1}$ Invasive and non-invasive diagnostic investigations, as well as necropsy studies showed that scleroderma cardiac involvement is one of the most frequent visceral complication that can affect the overall prognosis of the disease. ${ }^{1-8}$ More interestingly, heart involvement can appear independently from other typical complications of SSc. ${ }^{8}$ Although the myocardial fibrosis is a frequent pathological finding, cardiac involvement can be clinically silent. In particular, congestive heart failure and myocardial infarction are uncommon, while conduction system and rhythm disturbances were frequently recorded. ${ }^{1-6}$ Necropsy studies $^{8-11}$ demonstrated that scleroderma hearts without significant coronary lesions are frequently characterised by patchy myocardial fibrosis in both ventricles, resulting from collagen deposition in the areas of myocyte necrosis. Moreover, the presence of contraction band necrosis suggested that myocardial microcirculation disturbances, the so called "myocardial Raynaud' phenomenon" are responsible for the myocardial fibrosis, as indirectly supported by in vivo clinical investigations. ${ }^{12-17}$

Quantitative analysis of two dimensional echocardiographic (2D echo) pattern, or "texture", represents a novel approach for myocardial tissue characterisation. ${ }^{18}{ }^{19}$ This technique was able to identify acute ischaemic and contused myocardium lesions in animal models, ${ }^{18} 19$ as well as in human heart diseases including amyloid, diabetes, hypertrophic cardiomyopathies, myocarditis, myocardial ischaemia, and more recently hypertensive cardiopathy. ${ }^{20-25}$ The aim of this study was to detect changes in myocardial structure by means of ultrasonic videodensitometric analysis in a series of SSc patients without clinical evidence of ventricular disfunction or other complications responsible for secondary heart involvement. 
Table 1 Demographic and clinicoserological data of 35 SSc patients

\begin{tabular}{lll}
\hline & & Range \\
\hline Age (y; mean (SD)) & $48.6(11)$ & $22-65$ \\
M/F ratio & $3 / 32$ & \\
Disease duration (y; mean (SD)) & $8.3(6.0)$ & $1-26$ \\
Raynaud's syndrome & $97 \%$ & \\
Calcinosis & $26 \%$ & \\
Telangectasias & $86 \%$ & \\
Oesophagus & $74 \%$ & \\
Lung involvement & $88 \%$ & \\
Renal involvement & $0 \%$ & \\
Autoantibodies & & \\
ANA & $82 \%$ & \\
ACA & $40 \%$ & \\
anti-Scl70 & $37 \%$ & \\
\hline
\end{tabular}

ANA = anti-nuclear; $\mathrm{ACA}=$ anti-centromere antibodies.

\section{Methods}

Thirty five SSc patients (three males, aged 48.6 (11) years, mean (SD), range 22-65) and a sex and age matched group of 25 control subjects (two males; mean age 45.6 (10), range 25-63) without known systemic, immunological, and cardiovascular diseases were selected for the study.

All patients met the American College of Rheumatology (formerly, the American Rheumatism Association) 1980 preliminary criteria for the classification of SSc ${ }^{26} ; 31$ patients had a limited cutaneous involvement (distal to elbows and knees) and four the diffuse variant. ${ }^{6}$

Patients were consecutively recruited at the routine ambulatory control visits according to the following inclusion criteria: age $\leqslant 65$ years; absence of heart failure on clinical examination with normal chest radiographs and left ventricular systolic function at echocardiographic examination; absence of myositis, arterial hypertension, renal involvement, diabetes, and any other systemic diseases. In all cases ECG maximal exercise stress test was negative.

All subjects had given their informed consent to enter the study, which was approved by our Institutional Ethical Committee.

At the time of the study, all patients underwent a wide evaluation of the signs and symptoms of SSc; namely, extent of the cutaneous involvement (skin score), ${ }^{27}$ presence of telangiectasias, cutaneous calcinosis (radiographs), hypermelanosis, cutaneous ulcers, Raynaud's phenomenon, arthritis, oesophageal involvement (radiographic hypomotility with

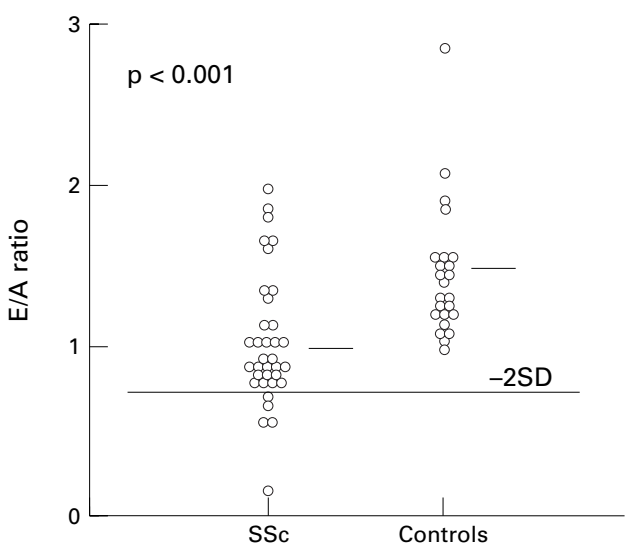

Figure 1 E/A ratio individual plot. -2 SD: 2 standard deviations under the mean value of control subjects. or without dysphagia), and nephropathy (proteinuria $>300 \mathrm{mg} / 24$ hours and/or serum creatinine $>1.2 \mathrm{mg} / \mathrm{dl}$ ). The presence of lung involvement was carefully investigated as previously reported ${ }^{5}$; in particular, all patients underwent a physical examination, chest radiograph (heart size, lung fibrosis, size of main pulmonary arteries), pulmonary function testing (total lung capacity, forced vital capacity, forced expiratory volume in one second), diffusing lung capacity for carbon monoxide (DLco).

Nailfold capillary examination was performed using a Leitz microscope and interpreted according to the procedures of Maricq et al. ${ }^{28}$ Capillaroscopic changes included diffuse tortuosity, enlarged capillary loops, reduced number of capillaries (total score 4.5).

In all patients the following serological markers were detected using standard techniques: anti-nuclear (ANA) antibodies by indirect immunofluorescence on rat liver at a dilution of serum of 1:20, and on Hep 2 cell line at a dilution of 1:40; anti-centromere antibody $(\mathrm{ACA})^{29}$; anti-extractable nuclear antigen (ENA) antibodies, including anti-Scl70, -Sm, -RNP, -SSB, -SSA, -PCNA, -SL and Jo1. ${ }^{30} 31$

CARDIOLOGICAL INVESTIGATIONS

In all patients resting and exercise ECG, 24 hour Holter ECG monitoring were performed and evaluated as previously described. ${ }^{4}$

Conventional echo Doppler analysis

Conventional echocardiographic studies were performed with a Hewlett-Packard (Andover, Mass, USA) 77020A phased array sector scanner with a 2.5 or $3.5 \mathrm{MHz}$ transducer. Two dimensional images were obtained in the parasternal long axis and short axis views and apical two and four chamber views. Left ventricular diameters (EDD: left ventricular end diastolic diameter), end diastolic septal thickness (Sthd) and posterior wall thickness (PWthd) were also measured, according to the criteria of the American Society of Echocardiography. ${ }^{32}$ Left ventricular per cent fractional shortening was calculated as end diastolic diameter minus end systolic diameter divided by end diastolic diameter multiplied by 100. Left ventricular mass was calculated by the Devereux formula (Penn convention) ${ }^{33}$ and adjusted for body surface area (LVMi(BS)). Pulsed Doppler transmitral flow velocity profile was obtained from the apical four chamber view, and the sample volume was positioned just below the mitral valve leaflets. The following parameters were evaluated: peak $\mathrm{E}$ (peak transmitral flow velocity in early diastole); peak A (peak transmitral flow velocity in late diastole); E/A ratio. Video recordings of $M$-mode imaging measurements were analysed off line by an experienced echocardiographer, using the Hewlett-Packard software of analysis. All recordings were analysed on two separate occasions within one week, for intraobserver variability, to assess the reproducibility of these measures, as well as by a blinded investigator for interobserver variability. Both interobserver and intraobserver variability were minimal; 
Table 2 Conventional echo Doppler parameters

\begin{tabular}{llll}
\hline & $\begin{array}{l}\text { Systemic sclerosis } \\
(n=35) \text { mean }(S D)\end{array}$ & $\begin{array}{l}\text { Controls }(n=25) \\
\text { mean }(S D)\end{array}$ & $\begin{array}{l}\text { Analysis of variance } \\
p \text { Value }\end{array}$ \\
\hline EDD $(\mathrm{mm})$ & $44.5(4.1)$ & $45.7(3.6)$ & 0.21 \\
FS $(\%)$ & $39.2(6.4)$ & $40.7(6.2)$ & 0.31 \\
LA $(\mathrm{mm})$ & $32.4(5.9)$ & $31.3(4.0)$ & 0.39 \\
Sthd $(\mathrm{mm})$ & $9.3(1.1)$ & $8.2(1.1)$ & 0.02 \\
Pwthd $(\mathrm{mm})$ & $8.9(1.2)$ & $8.0(1.2)$ & 0.06 \\
LVMi $(\mathrm{BS})\left(\mathrm{g} / \mathrm{m}^{2}\right)$ & $83.7(20.2)$ & $81.5(19.8)$ & 0.18 \\
Peak E (m/sec) & $0.69(0.22)$ & $0.80(0.21)$ & 0.07 \\
Peak A (m/sec) & $0.70(0.12)$ & $0.57(0.13)$ & 0.007 \\
E/A ratio & $1.03(0.42)$ & $1.44(0.28)$ & 0.001 \\
\hline
\end{tabular}

EDD = end diastolic diameter; FS = fractional shortening; Sthd = diastolic interventricular septum thickness; Pwthd $=$ diastolic posterior wall thickness; LVMi $(B S)\left(\mathrm{g} / \mathrm{m}^{2}\right)=$ left ventricular mass index (body surface indexed); LA = left atrium dimension.

coefficient of variation regarding $\mathrm{LV}$ mass values and other conventional echocardiographic parameters ranged from 7 to $9 \%$ for interobserver, and from $8 \%$ to $11 \%$ for intraobserver variability. Measurements were derived from the average of at least five consecutive cardiac cycles; in particular the diastolic parameters were also repeatedly measured to avoid the breathing induced changes.

\section{Quantitative textural analysis (videodensitometry)}

For a reproducible sampling of textural ultrasonic parameters, the gain settings and the gain compensation profiles were adjusted to obtain an apparently uniform myocardial brightness. The gray scale transfer function was adjusted to be linear for the entire video signal range, and no reject, no enhancement of dynamic range were used (18-19) with a 25-30 dB amplification at a depth of $18 \mathrm{~cm}$. The optimal echocardiographic images were directly transferred from the echocardiograph to a calibrated video digitisation system. Images were converted into $256 \times 256$ pixels of 256 gray levels each $(0=$ black, $255=$ white $)$ with eight bits of intensity range, by a commercially real time videodigitiser (Tomtec Imaging Systems, Boulder, Colorado, USA). One cardiac cycle (R-R wave) was automatically divided in 12 frames, independently by heart rate, and the images corresponding to the end diastolic and end systolic phases, all in left parasternal long axis view, were selected with an optimal visualisation of the interventricular septum and of the left ventricular posterior wall. Using an interactive computer program the region of interest, always the same size $(32 \times 42$ pixels $)$, was placed at mid-septum and mid-posterior wall level, both in end systole and end diastole, including only the myocardium and excluding the endocardial and epicardial specular echoes to avoid areas of echo dropouts and obvious

Table 3 Ultrasonic textural data (first order analysis)

\begin{tabular}{llll}
\hline & $\begin{array}{l}\text { Systemic sclerosis } \\
\text { mean (SD) }\end{array}$ & Controls mean (SD) & p Values \\
\hline MGLed (septum) & $65.8(24.2)$ & $89.9(13.5)$ & 0.001 \\
MGLes (septum) & $77.2(18.4)$ & $58.0(14.7)$ & 0.001 \\
CVI (\%) (septum) & $-23.7(30.3)$ & $35.9(20.9)$ & 0.0001 \\
MGLed (p. wall) & $78.1(27.6)$ & $92.3(15.8)$ & 0.01 \\
MGLes (p. wall) & $88.5(28.7)$ & $44.9(18.4)$ & 0.005 \\
CVI (\%) (p. wall) & $-20.5(32.7)$ & $50.9(20.6)$ & 0.0001 \\
\hline
\end{tabular}

MGLEd = Mean gray level (background corrected) in end diastole; MGLEs = mean gray level (background corrected) in end systole; CVI = cylic variation index. artefacts. For each region of interest, a histogram of the echocardiographic gray level distribution was generated, plotting the gray level distribution on the abscissa and the frequency of the occurrence on the ordinate. The mean gray level in each cavity region (background signal) was subtracted from the absolute mean gray level obtained at myocardial level obtaining both endsystolic mean gray level (MGLes) and end diastolic mean gray level (MGLed). Cyclic variation index of gray level amplitude was also calculated according to the formula: (MGLed - MGLes) / MGLed $\times 100) \cdot{ }^{34}$ All measurements were obtained from the average of five consecutive cardiac cycles. Coefficient of variation regarding MGL, both at end systolic and end diastolic values, ranged from $8 \%$ to $10 \%$ for interobserver, and from $9 \%$ to $12 \%$ for intraobserver variability.

\section{STATISTICAL ANALYSIS}

Continuous variables were expressed as mean (SD). Differences were tested for significance by unpaired Student's $t$ test. Upper and lower 95\% confidence limits for each variable were calculated from the two tails of the Student's $t$ test distribution by using the following formulas: mean $+2.042 \times \mathrm{SD}$, and mean $-2.042 \times$ $\mathrm{SD}$; where 2.042 represents the value of $t_{\alpha / 2}(\alpha$ is the probability value equal to $0.05: \mathrm{t}_{0.025}$ ) for a degree of freedom included between 30 and 60. Relations between videodensitometric and two dimensional echocardiographic measurements were expressed in terms of linear regression analysis. A p value $<0.05$ was considered significant.

\section{Results}

Table 1 shows the clinicoepidemiological and serological findings of 35 SSc patients. Among clinical manifestations lung involvement, Raynaud's phenomenon, and teleangectasias were the most frequently found. In all patients the presence of one or more serum autoantibodies was recorded.

Ambulatory 24 hour Holter ECG monitoring showed the presence of significant arrhythmias (premature ventricular complexes $\geqslant$ $200 / 24$ hours) in four of 35 SSc patients. In our group of SSc patients no conduction disturbances were detected during ECG Holter monitoring. The four patients with significant premature ventricular complexes, showed a clear cut abnormal ultrasonic textural pattern.

On the whole, the main values of echocardiographic parameters were comparable in both SSc patients and controls (table 2). In particular, mean values of fractional shortening, which represents a global index of systolic function, were comparable in both groups; similarly, left ventricular mass (body surface indexed) did not show significant differences between patients and controls.

Of the Doppler parameters, evaluating left ventricular diastolic filling, late peak flow (peak A) velocity was significantly higher in SSc patients than controls $(\mathrm{p}<0.007$, table 2$)$, while early peak flow (peak E) was reduced, but not significantly, if compared with normal controls. The E/A ratio was significantly lower in SSc 

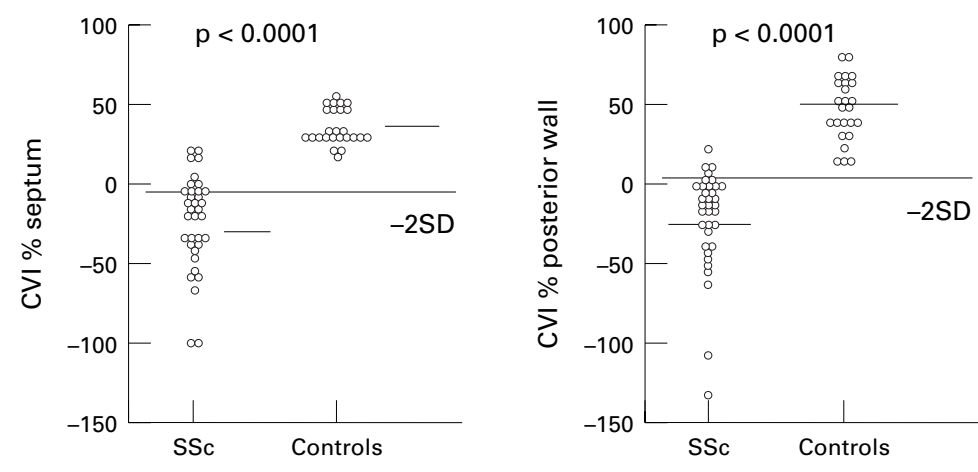

Figure 2 Cyclic variation index (CVI) at mid-septum and at mid-left ventricular posterior wall level for both systemic sclerosis $(S S c)$ and controls. $-2 S D: 2$ standard deviations under the mean value of control subjects.

patients than in normal subjects $(\mathrm{p}<0.001)$. When individual patients' analysis was performed, E/A ratio was under the normal range (95\% confidence intervals) in only five of 35 (15\%) of patients (fig 1).

Table 3 shows the videodensitometric parameters, in particular the mean gray level of the septum and of the posterior wall, both at end systole and end diastole. The mean values of CVI for both septum and posterior wall were significantly lower in SSc patients than controls (table 3, and fig 2; p<0.0001). In SSc patients the same parameter was abnormally reduced $(<95 \%$ confidence intervals) in $80 \%$ of cases
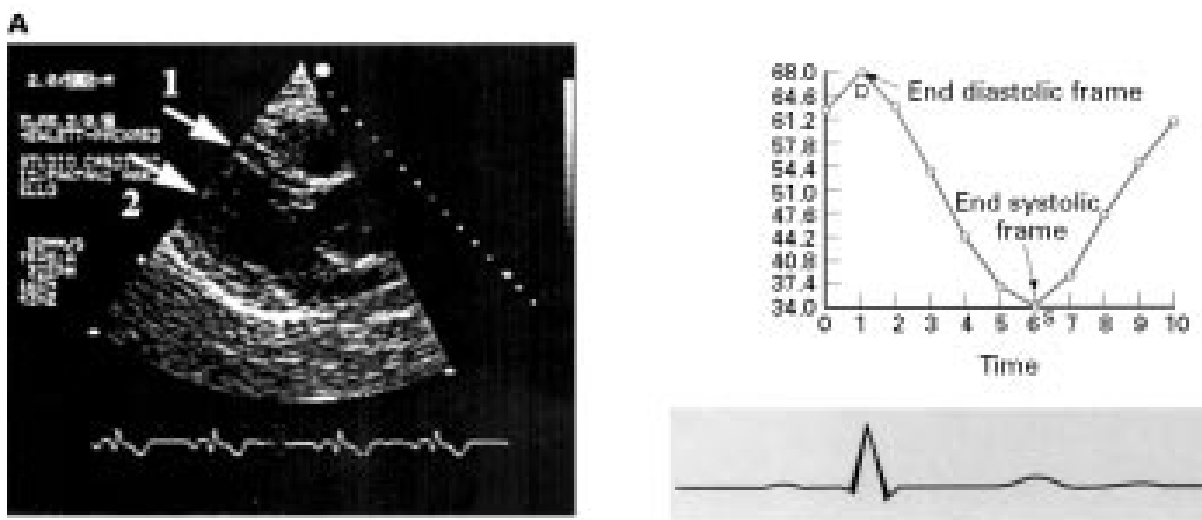

(28 of 35 ) for the septum and in $88 \%$ (31 of 35 ) for posterior wall, respectively.

Figure 3 compares the digitised 2D echo images of the left ventricle and the diagrams of echo intensity, in a selected region of the septum during one cardiac cycle, in one normal subject and one SSc patient. The mean gray values were totally changed in the patient compared with the normal subject; in particular, in the SSc patient the echo intensity values, during the cardiac cycle, showed a paradoxical behaviour.

No significant correlations were found between CVI and septum or posterior wall thickness, LVMi, FS\%, and diastolic Doppler transmitral flow parameters (peak E, peak A, E/A ratio).

Finally, no significant correlations were detected between epidemiological (age, sex, disease duration), clinical (extent of cutaneous involvement, nailfold capillaroscopy changes, skin calcinosis, lung involvement) and serological features (anti-centromere, anti-Scl70 antibodies) and textural videodensitometric findings. Interestingly, during the time period after this study one patient died because of severe cardiopulmonary involvement. This patient was one of those with more pronounced videodensitometric changes (CVI septum: $-102.7 \%)$

B
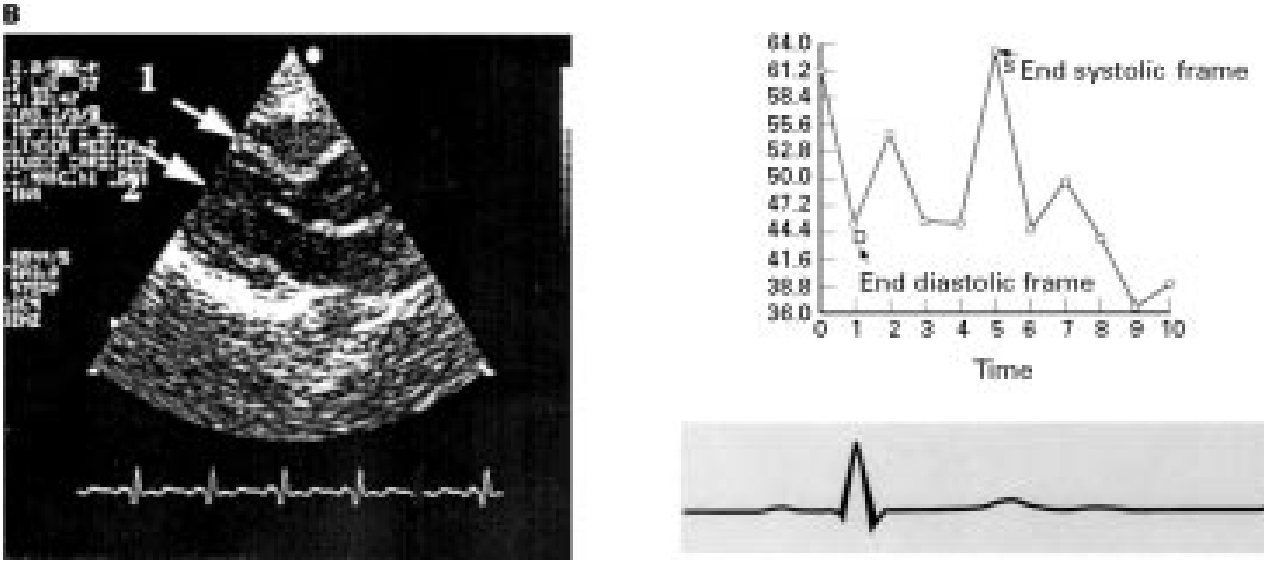

Figure 3 Comparison between digitised 2D echo images of the left ventricle (left side) and the diagrams of echo intensity variations (right side), in a selected region of the septum during one cardiac cycle, in one normal subject $(A)$ and one $S S c$ patient (B). Arrow 1 indicates the interventricular septum: the patient shows a clear cut increase in echo intensity suggesting the presence of fibrosis; arrow 2 indicates the left ventricular cavity for background subtraction. In the SSc patient the diagram of echo intensity variations shows a paradoxical behaviour compared with the normal subject. 


\section{Discussion}

Heart involvement in SSc patients is often clinically occult and its detection depends, at least in part, on the sensitivity of the diagnostic methods used. It has been estimated that scleroderma heart disease is clinically evident in only $20-25 \%$ of cases; in these people the prognosis becomes severe and a $70 \%$ of mortality at five years occurs. ${ }^{2}$ The discrepancy between the percentage of symptomatic heart involvement and necropsy scleroderma myocardial fibrosis $(30 \%-81 \%)^{2-12}$ should be improved by the introduction of more sensitive, possibly non-invasive, diagnostic tools.

This study demonstrated that myocardial tissue changes were detectable in the large majority of SSc patients $(88 \%)$ by means of ultrasonic videodensitometric analysis. In particular, the CVI, which is the expression of the intrinsic myocardial structural function was significantly lower, for both the septum and posterior wall, in scleroderma patients than in age matched controls. These findings can be the expression of preclinical heart abnormalities; in fact, all SSc patients had a normal left ventricular systolic function as well as a normal left ventricular mass. Coronary artery disease and other disorders responsible for myocardial damage - that is, arterial hypertension, diabetes, and other cardiomyopaties have also been excluded in the patients in this series. Moreover, left ventricular diastolic function was seldom impaired; actually, a change in E/A ratio was detected in only $15 \%$ of patients; this is particularly true as our age and sex matched control group showed a mean (SD) value of E/A ratio comparable with that reported by other authors. ${ }^{35}$ The diastolic change observed in SSc patients mainly regards the relaxation phase, resulting in an increase of atrial contribution to filling (peak A) and a decrease of early diastolic filling (peak E). ${ }^{37}$ Left ventricular diastolic function depends on the complex interaction of ventricular isovolumic relaxation, diastolic filling, and compliance. ${ }^{37}$ Abnormalities of the Doppler transmitral profile have been described in a large number of cardiac diseases; unfortunately, there is a wide overlap of individual data points between patients and controls, which prevents the routine evaluation of diastolic function in individual patient by Doppler echocardiography. ${ }^{38}$ The rarity of diastolic changes and much more the absence of systolic left ventricular dysfunction in our SSc patients emphasise the clinical relevance of myocardial tissue changes detected by videodensitometry.

Several clinical studies focusing on the heart involvement in SSc patients have been performed by means of traditional echo Doppler, which showed various abnormalities - that is, right ventricular enlargements, concentric left ventricular hypertrophy with asymmetrical septal hypertrophy, impaired shortening fraction or hyperdynamic systolic function, diastolic functional abnormalities, left atrial enlargement, pericardial effusion. ${ }^{2}$ On the whole, echocardiographic left ventricular systodiastolic myocardial dysfunction have been detected in a limited percentage of patients, ranging from $11 \%$ to $32 \% .^{2}$ In contrast, high incidence $(71 \%-100 \%)$ of thallium perfusion abnormalities was detected in SSc patients. ${ }^{13}{ }^{14} 17$ The coronary angiography usually shows normal epicardial coronary arteries, ${ }^{2}{ }^{13}$ while coronary reserve was often impaired ${ }^{15}{ }^{16}$; these findings suggested a prevalence of microcirculatory disturbances. ${ }^{13}$ The scintigraphic abnormalities are strikingly associated with pathological lesions, even in the absence of left ventricular dysfunction. ${ }^{2}$ Similarly, the high incidence of videodensitometric abnormalities observed in this study could reflect the presence of myocardial structural changes, detectable at a preclinical level.

The myocardial involvement in SSc has been reported in up to $81 \%$ of SSc patients at necropsy. ${ }^{9}{ }^{10}$ The main pathological findings were represented by extensive focal myocardial fibrous replacements that are prevalent in perivascular areas ${ }^{910}$ and contraction band necrosis, a pattern similar to that observed after reperfusion following transient coronary occlusion. ${ }^{12}$ Other necropsy findings, namely focal infiltrates of round cells and thickening of smaller coronary vessels, were less frequently represented. ${ }^{8}$ However, the exact mechanism leading to the myocardial fibrosis in SSc remains unknown. The paucity of structural vascular disease in the presence of contraction band necrosis could be explained by different pathogenetic hypotheses; namely, functional abnormalities such as small vessel vasospasm, the so called "intramyocardial Raynaud's phenomenon", or structural intramural microcirculatory changes. The presence of exercise and cold induced thallium perfusion abnormalities ${ }^{13} 1417$ and abnormal coronary vasodilator reserve,${ }^{15}{ }^{16}$ together with the above pathological findings suggested that scleroderma myocardial changes are the consequence of various cofactors. Among these myocyte replacement by collagen and coronary vasospasm seem to be the main pathogenetic factors of the myocardial fibrosis. The possible contribution of small artery obliterative disease and the damage of coronary capillary is suggested by in vivo physiological studies such as thallium perfusion changes and impaired coronary reserve $\mathrm{e}^{13-17}$; however, preliminary necropsy studies did not confirm this hypothesis. $^{2}$

The high frequency of myocardial texture changes detected in SSc patients is particularly significant if compared with that recorded in other disorders, ${ }^{20-25}$ in which comparable changes, but to a lesser degree, were found.

In normal subjects a cardiac cycle dependent variation in ultrasound signals within the myocardium was detectable: maximum values occurred at end diastole, minimal values at end systole. $^{39}{ }^{40}$ These cyclic changes could be related to intrinsic contractile function, ${ }^{41-43}$ distinct from myocardial wall thickening, although the physiological basis of this index is not fully understood and it is almost certainly multifactorial. ${ }^{44}$ For the first time we applied the videodensitometric approach to study the health of SSc patients. ${ }^{20-25}$ 
The significantly lower values of CVI are essentially because of the abnormal increase of the mean gray level at the end systole, than in controls; the reduction of CVI could mainly be related to scleroderma myocardial fibrosis, which causes an increase in acoustic impedance. This finding is mirrored by the high percentage of patients with myocardial fibrosis, the hallmark of scleroderma heart involvement, detected in necropsy studies. ${ }^{8-11}$ More interestingly, the results of this study further support the demonstration of myocardial fibrosis at necropsy and contraction band necrosis even in patients without clinical evidence of myocardial dysfunction. ${ }^{10}$ This latter condition was indirectly confirmed in our patients by the lack of correlation between myocardial changes and patients' age and disease duration as well as other prognostically relevant clinicoserological manifestationsthat is, skin sclerosis extent, capillaroscopic changes, lung involvement, and serum antiScl-70 antibody. In the light of this, it could be supposed that primary myocardial involvement in SSc patients represents a precocious event, characterised clinically by infrequent evolution to overt heart failure and more frequently by conduction system abnormalities, autonomic cardiac neuropathy, and often severe, life threatening arrhythmias. ${ }^{2-48} 4546$

In conclusion, videodensitometry represents a non-invasive, feasible ultrasound tissue characterisation method, able to early detect myocardial changes in SSc patients. The physiological and clinicoprognostic relevance of ultrasonic myocardial abnormalities in scleroderma patients needs to be further evaluated by prospective clinicopathological studies. In this respect, the patient with rather marked videodensitometric changes who died during the follow up suggests a potential prognostic relevance of such myocardial changes. Moreover, the analysis of scleroderma heart disease, mainly at a preclinical level, can give us new insights in the important field of primary cardiomyopaties.

1 Medsger TA Jr. Systemic sclerosis (scleroderma), localized scleroderma, eosiniphilic fascitis, and calcinosis. In: $\mathrm{McCa}-$ rty DJ, ed. Arthritis and allied conditions. Philadelphia: Lea and Febiger, 1118-65.

2 Deswal A, Follansbee WP. Cardiac involvement in scleroderma. Rheum Dis Clin North Am 1996;22:841-61.

3 Clements Pl, Furst DE, Cabeen W, Tashkin D, Paulus HE, Roberts N. The relationship of arrhythmias and conduction disturbances to other manifestations of cardiopulmonary disease in progressive systemic sclerosis (PSS). Am J Med 1981;71:38-46.

4 Ferri C, Bernini L, Bongiorni MG, Levorato D, Viegi G, Bravi $\mathrm{P}$, et al. Noninvasive evaluation of cardiac dysrhythmias, and their relationship with multisystemic symptoms, mias, and their relationship with multisystemic symptoms, in progressive system

5 Ferri C, Bernini L, Cecchetti R, Latorraca A, Marotta G, Passero G, et al. Cutaneous and serological subsets of systemic sclerosis. J Rheumatol 1991;18:1826-32.

6 Steen VD, Powell DL, Medsger TA. Clinical correlations and prognosis based on serum autoantibodies in patient with systemic sclerosis. Arthritis Rheum 1988;31:196-203.

7 Roberts NK, Cabeen WR, Moss J, Clements PJ, Furst DE. The prevalence of conduction defects and cardiac arrhythmias in progre

8 James TN. De subitaneis mortibus: VIII. Coronary arteries and conduction system in scleroderma heart disease. Circulation 1974;50:844-56.

9 D'Angelo WA, Fries JF, Masi AT, Shulman LE. Pathologic observations in systemic sclerosis (scleroderma): A study of
58 autopsy cases and 58 matched controls Am J Med 58 autopsy case
$1969 ; 46: 428-40$
10 Follansbee WP, Miller TR, Curtiss EI, Orie JE, Bernstein RL, Kiernan JM, et al. A controlled clinicopathologic study
of myocardial fibrosis in systemic sclerosis (scleroderma). J of myocardial fibrosis in systemi

11 McWhorterJE IV, LeRoy EC. Pericardial disease in scleroderma (systemic sclerosis). Am J Med 1974;57:56671

12 Bulkley BH, Ridolfi RL, Salyer WR, Hutchins GM. Myocardial lesions of progressive systemic sclerosis: A cause of cardiac dysfunction. Circulation 1976;53:483-90.

13 Follansbee WP, Curtiss EI, Medsger TA, Steen VD, Uretsky BF, Owens GR, et al. Physiologic abnormalities of cardiac function in progressive systemic sclerosis with diffuse scleroderma. N Engl J Med 1984;310:142-8.

14 Alexander EL, Firestein GS, Weiss JL, Heuser RR, Leitl G, Wagner HN Jr, et al. Reversible cold-induced abnormalities in myocardial perfusion and function in systemic sclerosis. in myocardial perfusion and function

15 Kahan A, Nitenberg A, Foult JM, Amor B, Menkes CJ, Devaux JY, et al. Decreased coronary reserve in primary scleroderma myocardial disease. Arthritis Rheum 1985;28: $637-46$

16 Kahan A, Devaux J, Amor B, Menkes CJ, Weber S, Nitenberg A, et al. Nifedipine and Thallium-201 myocardial perfusion in progressive systemic sclerosis. $\mathrm{N}$ Engl J Med 1986;314:1397-402.

17 Steen VD, Follansbee WP. Thallium perfusion abnormalities predict survival and cardiac dysfunction in patients with systemic sclerosis. Arthritis Rheum 1992;35:S37.

18 Skorton DJ, Collins SM, Nichols J, Pandian NG, Bean JA, Kerber RE. Quantitative texture analysis in twodimensional echocardiography: application to the diagnosis of experimental myocardial contusion. Circulation 1983; of experiment 68 .

19 Skorton DJ, Melton HE, Pandian NG, Nichols J, Koyanagi S, Marcus ML, et al. Detection of acute myocardial infarction in closed-chest dog by analysis of regional twodimensional echocardiographic gray-level distributions. Circ Res 1983;52:36-44

20 Pinamonti B, Picano E, Ferdeghini EM, Lattanzi F, Slavich $\mathrm{G}$, Landini L, et al. Quantitative texture analysis in two-dimensional echocardiography: application to the diagnosis of myocardial amyloidosis. J Am Coll Cardiol 1989;14:666-71.

21 Chandrasekaran K, Aylward PE, Bsee SRF, Burns TL, Seward JB, Tajik AJ, et al. Feasibility of identifying amyloid and hypertrophic cardiomyopathy with the use of computerised quantitative texture analysis of clinical echocardiographic data. J Am Coll Cardiol 1989;13:832-40.

22 Lieback E, Hardouin I, Meyer R, Bellach J, Hetzer H. Clinical value of echocardiographic tissue characterisation in the diagnosis of myocarditis. Eur Heart J 1996;17:135-42.

23 Picano E, Faletra F, Marini C, Paterni M, Danzi GB, Lombardi $\mathrm{M}$, et al. Increased echodensity of transiently asynergic myocardium in humans: a novel echocardiographic sign of myocardial ischemia. J Am Coll Cardiol 1993;21:199207.

24 Di Bello V, Pedrinelli R, Giorgi D, Bertini A, Talarico L, Caputo MT, et al. Ultrasonic videodensitometric analysis of two different models of left ventricular hypertrophy: athlete's heart and hypertension. Hypertension 1997;29:93744

25 Di Bello V, Giampietro O, Matteucci E, Talarico L, Giorgi $\mathrm{D}$, Bertini A, et al. Ultrasonic videodensitometric analysis in type I diabetic myocardium. Coronary artery disease 1996;7:895-901.

26 Masi AT, Rodnan GP, Medsger TA Jr, Altman RD, D'Angelo, WA, Fries JF, et al. Preliminary criteria for the D'Angelo, WA, Fries JF, et al. Preliminary criteria for the
classification of systemic sclerosis (scleroderma). Arthritis classification of systemic

27 Kahaleh MB, Sultany GL, Smith EA, Huffstutter JE. A modified scleroderma skin scoring method. Clin Exp Rheumatol 1986;4:367-69.

28 Maricq HR. Widefield capillary microscopy: tecnique and rating scale for abnormalities seen in scleroderma and related disorders. Arthritis Rheum 1981;24:1159-65.

29 Moroi Y, Peebles C, Fritzler MJ, Steigerwald J, Tan EM. Autoantibody to centromere (chinetochore) in scleroderma sera. Proc Natl Acad Sci USA 1980;77:1627-31.

30 Bunn CC, Gharavi AE, Hughes GRV. Antibodies to extractable nuclear antigens in 173 patients with DNA binding positive SLE: an association between antibodies to ribonucleoprotein and Sm antigen observed by counterimmunoelectrophoresis. J Clin Lab Immunol 1982;8:13-17.

31 Venables PJV, Smith PR, Maini RN. Purification and characterization of the Sjögren's syndrome A and B antigens. Clin Exp Immunol 1983;54:731-8.

32 Sahn DJ, DeMaria A, Kisslo J, Weyman A. Recommendations regarding quantitation in M-mode echocardiography: results of a survey of echocardiographic measurements. Circulation 1978;58:1072-83.

3 Devereux RB, Lutas ME, Casale PN, Kligfield P, Eisemberg RR, Hammond IW, et al. Standardization of M-Mode echocardiographic left ventricular anatomic measurements. J Am Coll Cardiol 1984;4:1222-30.

34 Lythall DA, Gibson DG, Kushwaha S, Norell MS, Mitchell AG, Ilsley CJD. Changes in myocardial echo amplitude during reversible ischemia in humans. Br Heart J 1992;67: 368-76.

35 Wind BE, Snider AR, Buda AJ, O'Neill WW, Topol EJ, Dilworth LR. Pulsed Doppler assessment of left ventricular diastolic filling in coronary artery disease before and immediately after coronary angioplasty. Am J Cardiol 1987;59:1041-6. 
36 Bryg RJ, Williams GA, Labovitz AJ. Effect of aging on left ventricular diastolic filling in normal subjects. Am J Cardiol ventricular dias

37 Smith VE, Zile MR. Relaxation and diastolic properties of the heart. In: Fozzard HA, et al, eds. The heart and cardiovascular system. 2nd ed. New York: Raven Press, 1991 1353-67.

38 Marshall SA, Levine RA, Weyman AE. Echocardiography in cardiac research. In: Fozzard HA, et al, eds. The heart and cardiovascular system. 2nd ed. New York: Raven Press, 1991:745-838

39 Miller JG, Perez JE, Sobel BE. Ultrasonic characterisation of myocardium. Prog Cardiovasc Dis 1985;28:85-110.

40 Skorton DJ, Miller JG, Wickline A, Barzilai B, Collins SM, Perez JE Ultrasonic characterisation of cardiovascular tissue. In: Marcus ML Schelbert HR, Skorton DJ, Wolf GL, eds. Cardiac imaging. Philadelphia: WB Saunders, 1991:886-95.

41 Perez JE, Barzilai B, Wickline SA, Vered Z, Sobel BE, Miller JG. Quantitative characterization of myocardium with ultrasonic imaging. J Nucl Med Allied Sci 1988;32:149-57.
42 Angermann CE, Stempfle HU. Tissue characterization in myocardial disease. In: Roelantd JRTC, Sutherland GR, lliceto S, Linker D, eds. Cardiac ultrasound. London: Churchill Livingstone, 1992:419.

43 Wickline SA, Thomas III LJ, Miller JG, Sobel BE, Perez JE. A relationship between ultrasonic integrated backscatter and myocardial contractile function. J Clin Invest 1985;76: 2151-60.

44 Logan-Sinclair R, Wong CM, Gibson DG. Clinical application of amplitude rocessing of echocardiographic images. Br Heart J 1981;45:621-7.

45 Bulkley BH, Klacsmann PG, Hutchins GM. Angina pectoris, myocardial infarction, and sudden cardiac death with normal coronary arteries: A clinicopathologic study of nine patients with progressive systemic sclerosis. Am Heart J 1978;95:563-74.

46 Ferri C, Emdin M, Giuggioli D, et al. Autonomic disfunction in systemic sclerosis: time and frequency
domain 24 hour heart rate variability analysis. $\mathrm{Br} \mathrm{J}$ Rheumatol 1997;36:669-76. 